\title{
Multispectral Image Classification Based on the Bat Algorithm
}

Original Scientific Paper

\author{
Almas Ahmed Khaleel \\ University of Mosul, \\ College of Basic Education, Department of Mathematics \\ Mosul, Iraq \\ diamond-12@uomosul.edu.iq
}

\author{
Joanna Hussein Al-Khalidy \\ Ninevah University, \\ College of Electronic Engineering, Department of Computer and Informatics Engineering \\ Mosul, Iraq \\ joanna.abdulhakeem@uoninevah.edu.iq
}

\begin{abstract}
There are many traditional classification algorithms used to classify multispectral images, especially those used in remote sensing. But the challenges of using these algorithms for multispectral image classification are that they are slow to implement and have poor classification accuracy. With the development of technologies that mimic nature, many researchers have resorted to using intelligent algorithms instead of traditional algorithms because of their great importance, especially when dealing with large amounts of data. The bat algorithm (BA) is one of the most important of these algorithms. This study aims to verify the possibility of using the BA to classify the multispectral images captured by the Landsat-5 TM satellite image of the study area. The study area represents the Mosul area located in the Nineveh Governorate in northwestern Iraq. The purpose is not only to study the ability of the $B A$ to classify multispectral images but also to obtain a land cover map of this region. The BA showed efficiency in the classification results compared to Maximum Likelihood (ML), where the overall accuracy of classification when using the BA reached (82.136\%), while MLreached (79.64\%).
\end{abstract}

Keywords: Bat algorithm; Multispectral image; Classification; Land cover classification

\section{INTRODUCTION}

In recent years, algorithms based on swarm intelligence (SI) have grown rapidly and significantly. They are being used extensively in many mathematical and engineering applications as well as in digital image processing. The bat algorithm (BA) is one of the most widely used techniques for solving clustering problems especially in remote sensing images where traditional classification algorithms do not provide high accuracy [1].

The idea of the BA is taken from nature. When bats fly at night in search of food, they emit echoes to guide them and determine their correct path, thus avoiding any obstacles they might encounter in their path. During its flight, the bat uses a technology that is somewhat similar to a sonar. It listens to the echoes of the sound to guide it on its way. A bat's echolocation is determined by emitting high-frequency sound pulses through its mouth or nose and listening for the echo [2].
Many researchers have studied the bat-inspired algorithm and below are short summaries of their works:

In [3], the authors presented a bat-inspired algorithm (BA), which relied on the behaviour of echo sites, to select multiple thresholds for different levels using the principle of maximum entropy (MaxEnt). The experimental results obtained in this study indicated that the proposed BA algorithm could find multiple thresholds similar to the optimal ones that were determined using a comprehensive search approach. Compared with Cuckoo search(CS), the computational times show that the BA algorithm is superior to the CS algorithm.

In [4] the authors provide a comprehensive review of the new BA and its variants. In this review, the following three main features are summarized by analyzing the main features and updating the equations:

1- Frequency control: This feature may be similar to the one used for particle swarm optimization and harmony search. 
2- Auto Zoom: The BA can automatically zoom in on the area where promising solutions have been found. For this reason, he concluded that BA has a fast rate of convergence.

3- Parameter control: BA uses parameter control, which enables it to change parameter values while iterations continue.

In [5] the authors used the BA to solve the multi-level image threshold problem. From the results they obtained, they concluded that the BA outperformed all other tested algorithms.

In [6], the authors used the BA to improve the contrast of the grayscale fingerprint image. The purpose of the BA was to map gray level distributions of contrast enhancement endings.

In [7], the authors discussed in their paper a variety of research articles, most of which dealt with the possibility of modifying the BA and its available applications. Despite the characteristics and capabilities of this algorithm, the researchers argue, there are still some problems that require additional research, such as parameter tuning and parameter control in this algorithm. However, BA remains an efficient algorithm.

In [8], the authors used simulation experiments on six test functions to verify and improve the Binary Bat algorithm (BBA). According to the simulation results, it was found that the convergence velocity of the algorithm is relatively sensitive to the parameter setting of the algorithm used.

In the field of remote sensing, many methods are proposed to extract the important features from the image, the most important of which are the following:

In [9], the authors presented in their paper a method for extracting features of remote sensing images based on BA and natural chromatic aberration. Initially, the contrast of remote sensing images was improved by the BA. Then, the colour feature was extracted using normal chromatic aberration, and then the features were encoded in a binary system.

In [10], the authors used BA to solve the transport network design problem so as to get the best objective function value solution. BA outperformed all intelligent techniques such as Genetic Algorithms (GA) and, Ant Colony Optimization (ACO).

In [11], the authors explored BAT Algorithm (E-BA) with a 2-D histogram is based on multi-level image thresholding for effective picture thresholding which is achieved by maximising the Renyi entropy. E-BA was successfully tested on standard test pictures to demonstrate the algorithm's performance. The E- BA's obtained results were compared to Renyi entropy algorithms such as BAT and Particle Swarm Optimization (PSO). According to these comparisons, the algorithm (E-BA) has the highest fitness value among all the algorithms.
In [12], the authors presented a robust digital image watermarking scheme based on Static Wavelet Transform (SWT) using the Bat Optimization BA and the powerful Speedup (SURF) feature. The results showed that it is possible to achieve a targeted balance between watermark visibility and durability.

In [13] the authors presented a BA analysis based on the preliminary mathematical analysis and statistical comparisons of the first-hit time performance measurement distributions obtained on a test set of five carefully selected objective functions. The results show that BA is not an original contribution and does not generally outperform the PSO algorithm when making a fair comparison. Finally, the results indicate that the best BA version is a simple combination between PSO and simulated annealing.

In [14], the authors propose in their paper several Objective Bat algorithms (MaOBAT) that solve multiobjective optimization problems through effective approximation of Pareto approximation (PA) with high diversity and good convergence. A pilot study shows that MaOBAT works efficiently and has significant advantages over MaOPSO, SMPSO, and NSGA III.

Some previous studies in the field of multispectral and classification based on image processing were reviewed: Perumal and Bhaskaran compared the performance of different classifiers and found that the Mahalanobis classifier is superior even to the advanced classifiers. In terms of successful image classification, the simple and accurate workbook demonstrates the importance of considering the relationship between the data set and the classifier. Also, the researchers stated that additional studies are needed to improve the use of classifiers to increase the applicability of such methods [15].

In [16], Some researchers revealed the loss of vegetation cover for Nineveh Governorate by using remote sensing images based on the algorithm of an ant colony According to the results, the agricultural area and floodplain decreased from about $31 \%$ in 1987 to $11.2 \%$ in 2009 , while the origin of the early sandy plate and desert region increased from $42.7 \%$ to $49 \%$. Sand dunes also appeared in 2009 in about $26.47 \%$ of the overall study area.

In [17], the authors proposed a bat-based clustering algorithm. They used multispectral satellite images to help solve crop type classification problems. The performance of the proposed method is compared to that of three other techniques: K-mean clustering, GA, and PSO. Since BA successfully converges to optimal centres of mass, it was concluded that BA can be successfully applied to address crop type classification problems.

In [18], the authors presented a method for detecting buildings in densely populated urban areas by integrating data from the first pulsed laser scanner. The purpose of this method is to achieve a land cover classification.

In [19], the authors address the problem of multispectral satellite image classification of forest plants. 
Features were investigated based on wavelet transform and a classification method involving consideration of the significance of each feature.

In [20], the author investigated the neural network matrix classifier and its application in multispectral image classification. By the results obtained, the effectiveness of this classifier has been demonstrated compared to standard classifiers.

In [21], the authors optimized the K-mean algorithm according to the problems found in visual tagging when performing supervised classification. The weighting coefficient and $\mathrm{K}$-mean clustering method were combined to characterize the training samples, which reduced the subjective influence of visual interpretation on the classification results.

In [22], the authors proposed an automated approach to detecting water bodies based on the Dempster-Shafer theory of combining supervised learning with a specific property of water in the spectral range in a completely unsupervised context, thus providing valuable information for further land cover classification.

In [23], the authors presented a method of describing and classifying multispectral images that allows recognition of classes of different organisms was presented. The algorithm has been successfully applied to forest plants.

Using multispectral sensors, In [24], the authors proposed a new method for recording captured images and introduced a new fusion scheme that combines images differently for high and low frequencies.

In [25], the authors proposed a multispectral image classification based on an object-based active learning approach which is a novel method for object-based sampling.

In [26], the authors responded to the difficulty of detecting a change in multispectral images by proposing a methodology (GBF-CD) based on the merging of graphic data.

For our current study, Mosul city is the study area. It is located in Nineveh. Mosul is about $465 \mathrm{~km}$ to the north of Baghdad, as shown in Fig. 1 [24].

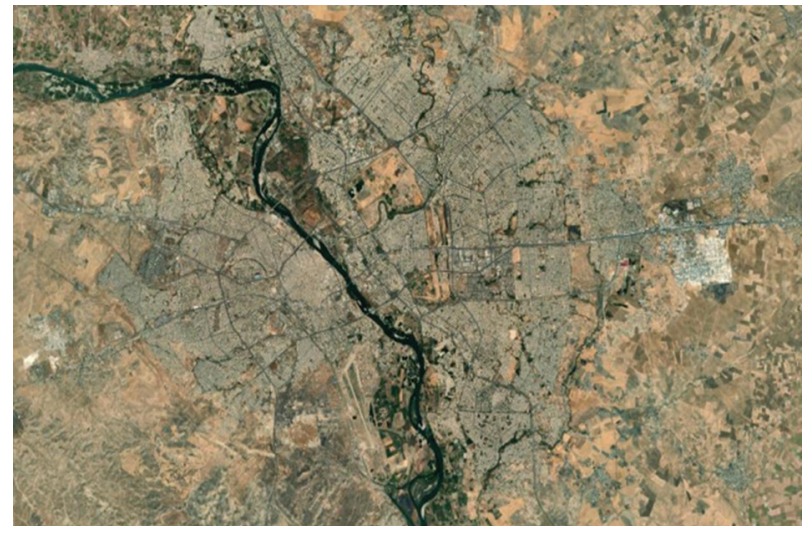

Fig. 1. Study Area
This study proposes a supervised classification method for multispectral image data based on the BA. The aim of this study is to use the BA for classifying multispectral satellite image datasets and compare their overall accuracy with the conventional image classification method.

The study organization is as follows: Section 1 presented an introduction; Section 2 presents the bat algorithm concept; Section 3 provides an image classification based on the BA. Section 4 discusses the proposed work and section 5 lists the results of the study.

\section{BAT ALGORITHM (BA)}

The bat algorithm (BA) is developed by Xin-She Yang in 2010 and it is established according to the following rules [3]:

Bats have an echolocation behaviour similar to that of microbats as they measure the distance and the difference between prey and background block. To locate a prey, bats fly indiscriminately in space vi at position $\mathrm{x}_{\mathrm{i}}$ with a frequency $f_{i}$ and loudness $A_{o}$. They may change the wavelength (frequency) of the pulses and the rate at which they release them based on the proximity of their prey. The loudness should range from a high positive value $A_{o}$ to a low constant value $A_{\min }$. As the number of samples grows, this rule can be changed. The following will be used to determine the new locations $x i(t)$ and velocities vi(t) [17]:

$$
\begin{gathered}
\mathrm{x}_{\mathrm{i}}(\mathrm{t}+1)=\mathrm{x}_{\mathrm{i}}(\mathrm{t})+\mathrm{v}_{\mathrm{i}}(\mathrm{t}+1) \\
\mathrm{v}_{\mathrm{i}}(\mathrm{t}+1)=\mathrm{v}_{\mathrm{i}}(\mathrm{t})+\left(\mathrm{x}_{\mathrm{i}}(\mathrm{t})-\mathrm{p}(\mathrm{t})\right) \cdot \mathrm{f}_{\mathrm{i}} \\
\mathrm{f}_{\mathrm{i}}=\mathrm{f}_{\text {min }}+\left(\mathrm{f}_{\text {max }}-\mathrm{f}_{\text {min }}\right) \cdot \beta
\end{gathered}
$$

When it is a regular random number the range will be $[0,1], f_{\min }=0, f_{\max }=1$ and $p(t)$ represents the current global best solution (location). When the bats' positions are updated, a random number is created. If the generated random number is more than the pulse emission rate $r_{i}$ a new solution is generated using a local random walk around the existing global best solution.

$$
\mathrm{x}_{\mathrm{i}}(\mathrm{t}+1)=\overrightarrow{\mathrm{p}}(\mathrm{t})+\varepsilon \overline{\mathrm{A}}(\mathrm{t})
$$

When the random number is $\varepsilon$, which is between [- 1 , $1]$, the population average loudness is $\bar{A}(t)$.

Furthermore, by regulating the loudness $A_{i}(t+1)$ and the pulse rate $r_{i}(t+1)$.

$$
\begin{gathered}
A_{i}(t+1)=\alpha A_{i}(t) \\
r_{i}(t+1)=r_{i}(0)[1-\exp .(-\gamma t)]
\end{gathered}
$$

The constants are $a$ and $\gamma$ while the initial values of loudness and pulse rate are $a>0, \gamma>0$. $A_{i}(0)$ and $r_{i}(0)$, respectively. The steps of the standard BA are described as follows: The first step: Initialize the position, velocity, and parameters for each bat, then use Equation to produce the frequency at random (3). 
The second step: Eq. (1) and Eq. (2) should be used to update each bat position and velocity.

The third step: Create a random number for each bat $(0<\operatorname{rand} 1<1)$. If rand $1 r_{i}(t)$, update the temp position and use Eq. (4) to get the fitness value for the corresponding bat.

The fourth step: Create a random number for each bat $(0$ rand 21$)$. If rand $2 A_{i}(t)$ and $f\left(x_{i}(t)\right) f(p(t))$, update $A_{i}(t)$ and $r_{i}(t)$ with Eq. (5) and Eq. (6), respectively.

The fifth step: Select each individual according to their fitness levels and save It.

The sixth step: If the requirements are met, the algorithm is complete; otherwise, proceed to the second step[11].

\section{Multispectral Image Classification using the BA}

Based on the above descriptions, the main steps of the BA consist of a single loop with some probabilistic switching during the iteration. Thus, the BA procedure is summarized in the following flowchart, as shown in Fig. 2.

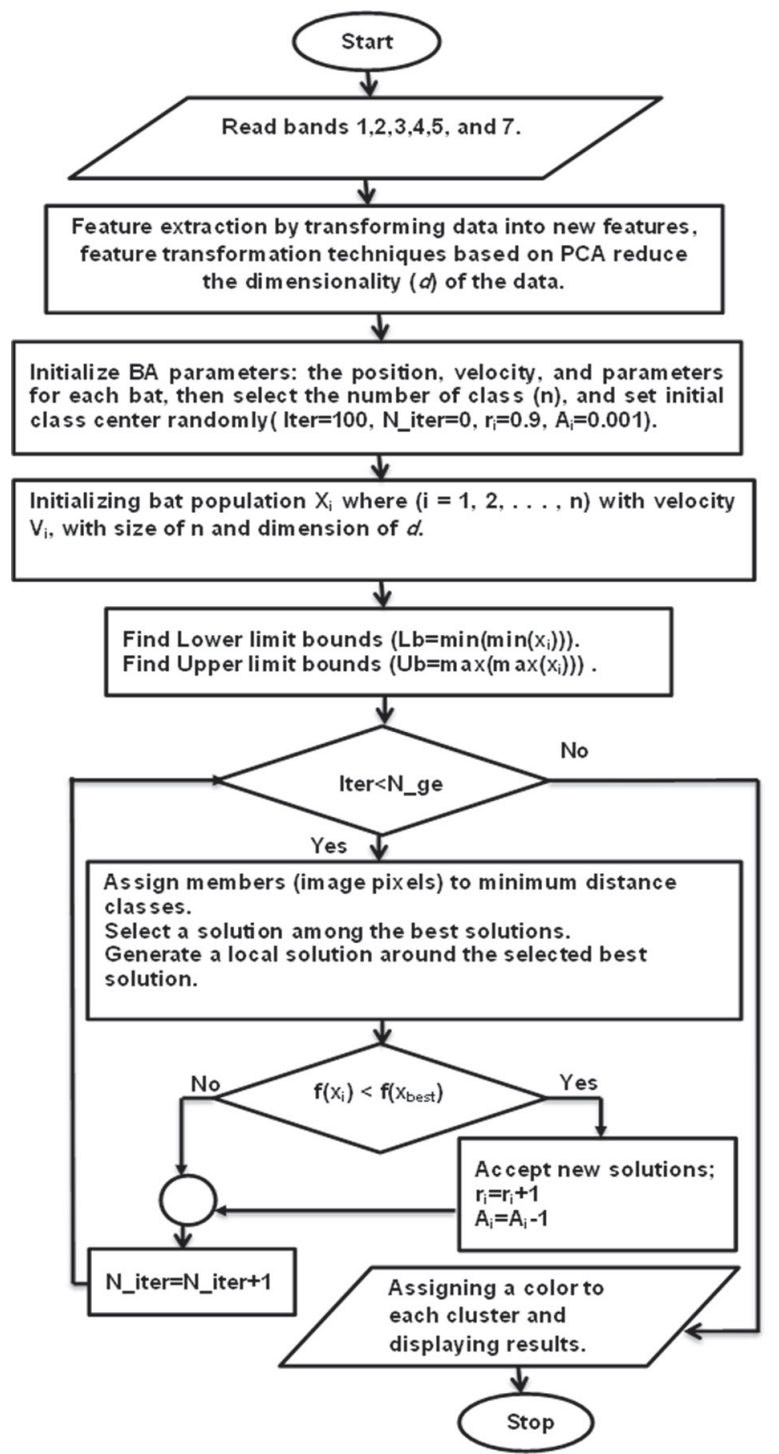

Fig. 2. BA flowchart

\section{PROPOSED METHODOLOGY}

Due to the large amount of data contained in multispectral images, dealing with the amounts of information contained in them is necessary. Dealing with it, though, is complicated. Unlike interpreting aerial imagery, these multi-spectral images do not allow for immediate object detection of the type of feature. As a result, a remote control is required. To help the user understand the features in the image, sensor data should be classified first, then processed by using various data optimization techniques. Depending on the classification algorithm used, this classification is a difficult task that needs careful validation of training samples. The classification algorithms in this study come in a variety of shapes and sizes. The simulation algorithm used is the BA.

Classification algorithms are gaining popularity in the field of remote sensing and land and land cover classification. Because of the complexity of extracting information from images and satellite data, the task of classifying images is a daunting task. But with the huge advances in swarm intelligence simulation in nature and artificial intelligence algorithms, it has helped solve classification problems. For this reason, the current study aims to verify the possibility of using the BA in land cover classification.

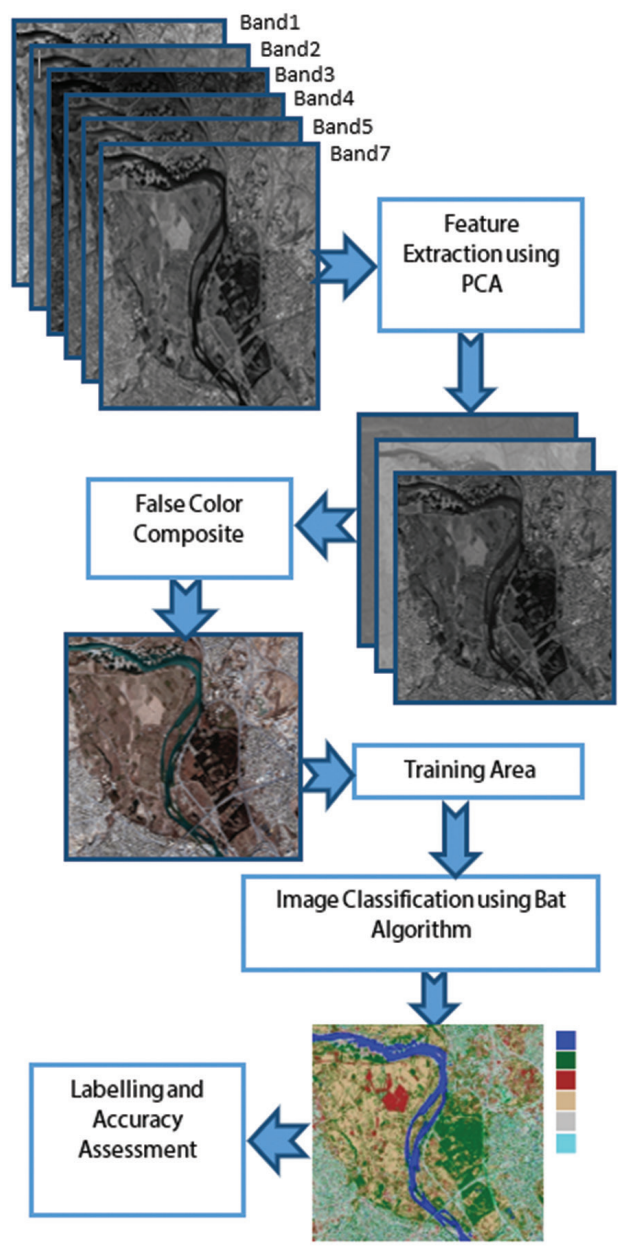

Fig. 3. Steps of multispectral image classification 
Figure 3 illustrates the basic steps that were applied in order to classify the multispectral images of the Mosul area in north western Iraq.

To control the amount of red, green, and blue in a colour image, three LANDSAT spectral bands were used to produce three-band composite images. The spectral range of vision of the human eye is roughly represented by the False-color composite images with band groups 7,4 , and 2; therefore these images appear close to what would be expected in a normal colour photograph. This phase is essential for determining the training area based on field information. A synthetic representation of a multi-spectral image is a false-colour image. The range numbers used for red, green, and blue in a given order are often used to identify the specific ranges used in tri-band combinations. So, an image that uses Band 7 will be assigned to red, Band 4 to green, and Band 2 to blue $(7,4,2)$. As shown in Fig. 4, the short wave complex of ranges 7, 4 and 2 represents the Tigris River in bluish-green, and the cultivated areas with different degrees of brown. The roads appear as straight, light lines, and so is the case with the rest of the other classes of land cover. The image classification process includes some basic steps such as optimization of multi-spectral images, feature extraction, use of the principal component analysis (PCA) algorithm to extract features, and reduction in the dimensions included in the classification. Instead of entering six images, three components are used (PCA1, PCA2, PCA3), as shown in Fig. 5.

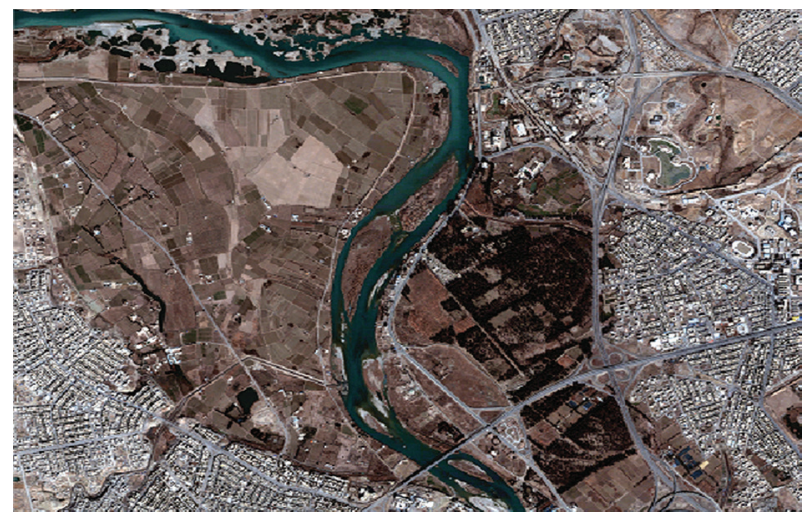

Fig. 4. False-color composite images with band groups $(7,4,2)$

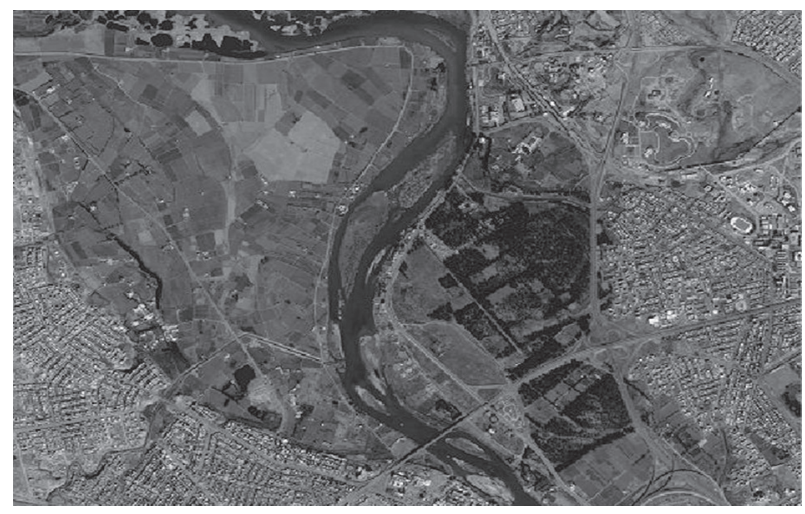

(a)

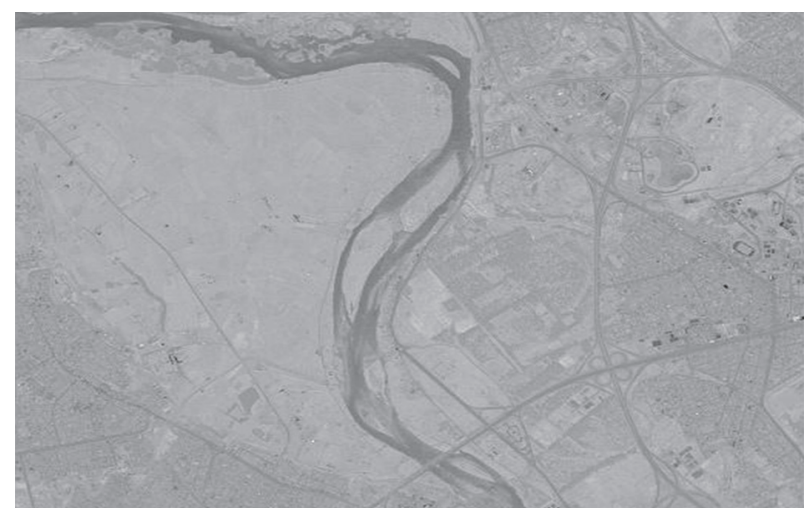

(b)

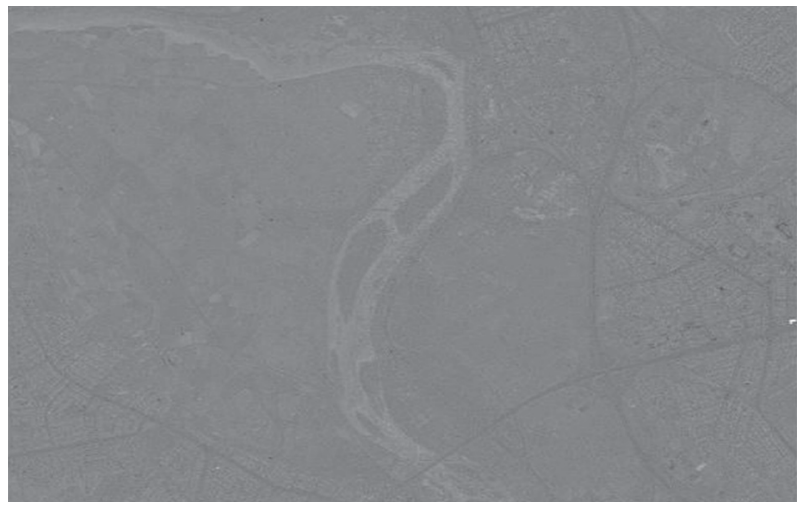

(c)

Fig. 5. Principal Component Analysis Results: (a) PCA1, (b) PCA2 and (c) PCA2

\section{RESULTS AND DISCUSSIONS}

The results of this study show the importance of relying on remote sensing data and Landsat satellite images with the sensor (TM) to detect discrimination and identification of land cover types in the study area, and the importance of using the BA programmed using Matlab to classify multispectral images.

To train the classifier, the training areas were selected from false images based on the field information and the GIS system available about the study area,. Finally, the BA is used to classify multispectral images by drawing on field information and generating colour land cover classification images. Fig. 6 illustrates the classification results.

The BA was used as a supervised classification that takes advantage of the collected training samples. Choosing spectral signatures for different classes of training areas is very important for training the supervised classifier. In this study, training areas were selected based on the false-colour composite in addition to the available field and geographic information about the study area. Since biased selection in supervised classification negatively affects classification accuracy, the accuracy of selection of training regions is important and that is why ERDAS IMAGINE was used to select training regions. Depending on the nature of the study area, the number of classes required to implement the BA was divided into six categories: Water, Agriculture, Barren, Grasslands, Roads/Birds, and Urban 
Areas. Fig. 6 and Fig. 7 show the result of the classification using the BA and ML after giving a label for each class and obtaining images of the land cover of the study area.

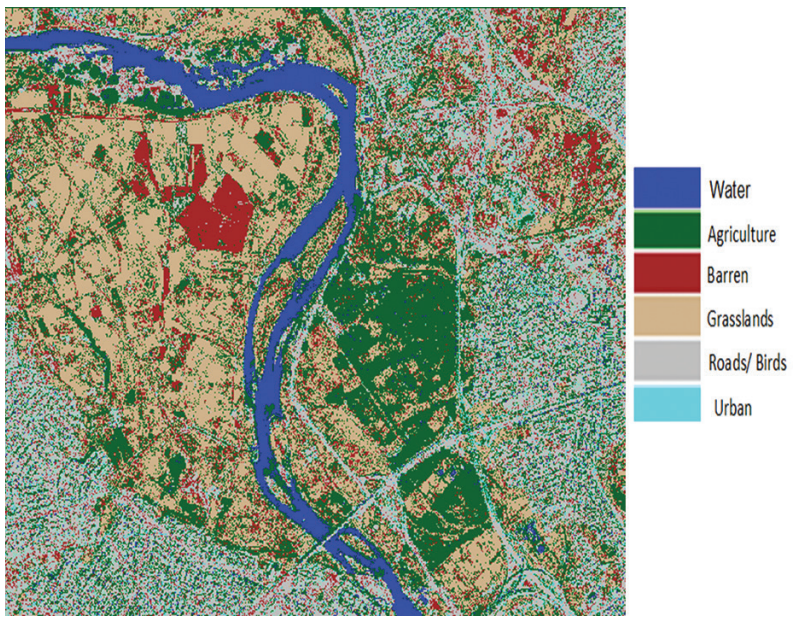

Fig. 6. BA Classification Result
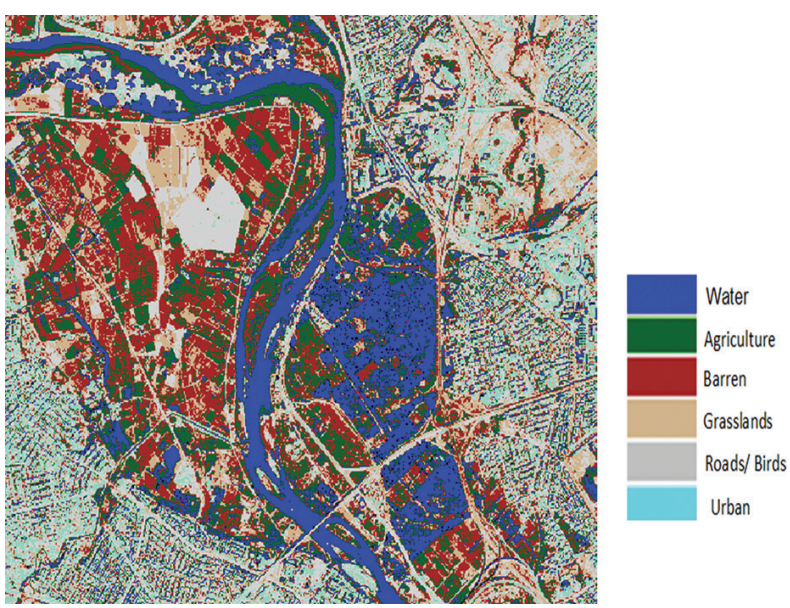

Fig. 7. ML Algorithm Classification Result

To create a land cover map, it is necessary to use a huge amount of data about the study area with the use of one of the classification algorithms. In this study, multispectral images were used, and they were classified using the BA. But the classification will not be useful to use unless accuracy is evaluated. As a result, the objectives of accuracy assessment are to evaluate the accuracy of the classification and document its usefulness so that it can be properly understood by others.

In most cases, accuracy assessment consists of two steps:

1. Collection of reference data. This data enables the identification of land cover classes at specific locations using a methodology independent of the data used for remote sensing classification. Reference data sources include high-resolution, remote-sensed spatial data, such as field or geographic information system information, and field survey measurements with GPS-recorded location.

$$
A C=C R / S R,
$$

where $A C$ denotes the correct rate. $C R$ represents the number of correctly classified pixel samples. SR represents the total number of pixels in each training class.

Omission error: pixels that belong to actual classes but aren't classified into them (e.g., 15 pixels which should have been classified as Grasslands, were classified as Agriculture).

After calculating the accuracy and error in the performance of the classifier for each class and calculating the final classification accuracy, the results were very acceptable as shown in Table 1 and Table 2 . When calculating the classification accuracy, we noticed that the correctly marked classes were the first, second and fourth classes, while the worst marked were the Fifth and sixth classes. But the overall accuracy of the BA classifier was better than the maximum likelihood algorithm, scoring $82.136 \%$ and $79.64 \%$, respectively.

Table 1. Accuracy Assessment (BA)

\begin{tabular}{|cccc|}
\hline Class No. & Class Name & Accuracy & Omission Error \\
\hline Class1 & Water & 96.08 & 3.92 \\
\hline Class2 & Agriculture & 87.61 & 12.39 \\
\hline Class3 & Barren & 84.4 & 15.6 \\
\hline Class4 & Grasslands & 87.51 & 12.49 \\
\hline Class5 & Roads/ Birds & 71.9 & 28.1 \\
\hline Class6 & Urban & 65.32 & 34.68 \\
\hline Overall Accuracy $=\mathbf{8 2 . 1 3 6 \%}$ & Overall Error $=\mathbf{1 7 . 8 6 3 \%}$ \\
\hline
\end{tabular}

Table 2. Accuracy Assessment (ML)

\begin{tabular}{|cccc|}
\hline Class No. & Class Name & Accuracy & Omission Error \\
\hline Class1 & Water & 90.16 & 5.71 \\
\hline Class2 & Agriculture & 84.83 & 10.9 \\
\hline Class3 & Barren & 83.5 & 18.99 \\
\hline Class4 & Grasslands & 88.33 & 10.32 \\
\hline Class5 & Roads/ Birds & 70.8 & 29.23 \\
\hline Class6 & Urban & 60.25 & 40.68 \\
\hline Overall Accuracy= 79.64\% & Overall Error= $\mathbf{2 4 . 8 0 5 \%}$ \\
\hline
\end{tabular}

\section{CONCLUSION}

In this study, the multispectral image was used to classify the land cover with acceptable accuracy. We used an unconventional classification method based on the BA to produce the land cover map. This study shows that relying on multispectral images is better than using single images; however, a method to extract the characteristics without losing information is needed. PCA was successfully used to extract the three compounds and enter them into the classification algorithm based on the BA and produce a land cover map for the study area. It has been concluded that the use of algorithms that mimic nature such as the BA is useful for classifying multispectral images. The BA proved successful and accurate in classifying multispectral images and producing a ground cover image of the study 
area. In the future, we will apply the BA to classify hyperspectral images. As well as using other types of different land cover features.

\section{ACKNOWLEDGEMENTS}

The authors are very grateful to University of Mosul / College of Basic Education, and Ninevah University/ College of Electronic Engineering for their provided facilities, which helped to improve the quality of this work.

\section{REFERENCES:}

[1] X.-S. Yang, "Swarm intelligence-based algorithms: A critical analysis", Evolutionary Intelligence. Vol. 7, 2014, pp. 17-28.

[2] S. Nandy, P. P. Sarkar, "Bat algorithm-based automatic clustering method and its application in image processing", Elsevier Ltd, 2016.

[3] A. Alihodzic, M. Tuba, "Bat Algorithm (BA) for Image Thresholding", Recent Researches in Telecommunications, Informatics, Electronics and Signal Processing, 2013, pp. 364-369.

[4] X.-S. Yang, "Bat algorithm: Literature review and applications", International Journal of Bio-Inspired Computation, Vol. 5, No. 3, 2013, pp. 141-149.

[5] A. Alihodzic, M. Tuba, "Improved bat algorithm applied to multilevel image thresholding", The Scientific World Journal, Vol. 2014, 2014.

[6] A. Bouaziz, A. Draa, S. Chikhi, "Bat Algorithm for Fingerprint Image Enhancement", Proceedings of the 12th International Symposium on Programming and Systems, Algiers, Algeria, 28-30 April 2015.

[7] S. L. Yadav, M. Phogat, "A Review on Bat Algorithm," International Journal of Computer Science and Engineering, Vol. 5, No. 7, 2017, pp. 39-43.

[8] X. X. Ma, J. S. Wang, "Optimized Parameter Settings of Binary Bat Algorithm for Solving Function Optimization Problems", Journal of Electrical and Computer Engineering, Vol. 2018, 2018.

[9] Y. Cao, Y. Xun, Y. Han, J. Chen, S. Wang, Z. Zhang, N. $\mathrm{Du}, \mathrm{H}$. Meng, “Feature Extraction of Remote Sensing Images Based on Bat Algorithm and Normalized Chromatic Aberration", IFAC-Papers On Line, Vol. 52, No. 24, 2019, pp. 318-323.

[10] S. Srivastava, S. K. Sahana, "Application of Bat Al- gorithm for Transport Network Design Problem", Applied Computational Intelligence and Soft Computing, Vol. 2019, 2019.

[11] V. Manohar, G. Laxminarayana, T. S. Savithri, "Image compression using explored bat algorithm by Renyi 2-d histogram based on multilevel thresholding", Evolutionary Intelligence, Vol. 19, No. 3, 2019, pp.1-11.

[12] A. Pourhadi, H. Mahdavi-Nasab, "A robust digital image watermarking scheme based on bat algorithm optimization and SURF detector in SWT domain", Multimedia Tools and Applications, Vol. 79, No. 29, 2020, pp. 1-25.

[13] I. Gagnon, A. April, A. Abran, "A critical analysis of the bat algorithm", Engineering Reports, July, 2020.

[14] U. Perwaiz, I. Younas, A. A. Anwar, "Many-objective BAT algorithm", PLoS One, Vol. 15, No. 6, 2020, pp. $1-20$.

[15] K. Perumal, R. Bhaskaran, "Supervised Classification Performance of Multispectral Images", Journal of Computing, Vol. 2, No. 2, 2010, pp. 124-129.

[16] Y. M. Abbosh, T. A. Khaleel, J. H. Al-Khalidy, “Vegetation Loss Detection of Nineveh Province Using Remote Sensing Images Based on Ant Colony Algorithm", Al-Rafidain Engineering Journal, Vol. 21, No.6, 2013, pp. 6-7.

[17] J. Senthilnath, S. Kulkarni, J. A. Benediktsson, X. S. Yang, "A Novel Approach for Multispectral Satellite Image Classification Based on the Bat Algorithm," IEEE Geoscience and Remote Sensing Letters, Vol. 13, No. 4, 2016, pp. 599-603.

[18] F. Rottensteiner, J. Trinder, S. Clode, K. Kubik, "Using the Dempster-Shafer method for the fusion of LIDAR data and multi-spectral images for building detection", Information Fusion, Vol. 6, 2005, pp. 283-300.

[19] A. I. Nazmutdinova, V. N. Milich, “Dependence of the results of classification of multispectral images of forest vegetation on wavelet-transform parameters", Optoelectronics, Instrumentation and Data Processing, Vol. 52, 2016, pp. 231-237.

[20] X. Fu, "Multispectral image classification based on neural network ensembles", Proceedings of the $8^{\text {th }}$ 
International Conference on Advanced Computational Intelligence, Chiang Mai, Thailand, 14-16 February 2016, pp. 275-277.

[21] X. Jing, S. Y. Chen, L. L. Fan, "Semi-supervised classification of multi-spectral images based on density", Proceedings of the $9^{\text {th }}$ International Conference on Digital Image Processing, Vol. 10420, Hong Kong, China, 2017, pp.1-6.

[22] A. M. Li Na, R. Estival, "An automatic water detection approach based on Dempster-Shafer theory for multi-spectral images", Proceedings of the $20^{\text {th }}$ International Conference on Information Fusion, Xi'an, China, 10-13 July 2017, pp. 1-8.

[23] A. I. Nazmutdinova, A. G. Itskov, V. N. Milich, “Description of the process of presentation and recognition of forest vegetation objects on multi- spectral space images", Pattern Recognition and Image Analysis, Vol. 27, 2017, pp. 105-109,.

[24] N. Ofir, S. Silberstein, D. Rozenbaum, Y. Keller, S.D. Bar, "Registration and fusion of multi-spectral images using a novel edge descriptor", Proceedings of the $25^{\text {th }}$ IEEE International Conference on Image Processing, Athens, Greece, 7-10 October 2018, pp. 1857-1861.

[25] T. Su, S. Zhang, T. Liu, "Multi-spectral image classification based on an object-based active learning approach", Remote Sensing, Vol. 12, No. 3, 2020, pp. 1-37.

[26] D. A. J. Sierra, H. D. B. Restrepo, H. D. V. Cardonay, J. Chanussot, "Graph-based fusion for change detection in multi-spectral images", arXiv:2004.00786, 2020 . 